\title{
Tecnura
}

\section{Reciclaje de EAFD a través de la lixiviación selectiva de zinc con soluciones de citrato: comparación con un ácido inorgánico}

\section{EAFD Recycling Through Selective Zinc Leaching with Citrate Solutions: Comparison with an Inorganic Acid}

\author{
Johana Borda (iD) 1 , Fabián López (iD)2 , Robinson Torres ${ }^{(D)} 3$, Mario Parra ${ }^{\text {(D) } 4}$
}

Fecha de Recepción: 16 de Noviembre de 2020

Fecha de Aceptación: 01 de Abril de 2021

Cómo citar: Borda., J. López., F. Torres., R. y Parra., M. (2021). Reciclaje de EAFD a través de la lixiviación selectiva de zinc con soluciones de citrato: comparación con un ácido inorgánico. Tecnura, 25(69), 131-149. https:/ / doi.org/10.14483/22487638.15767

\section{Resumen}

Contexto: Los polvos de acería (electric arc furnace dust, EAFD), son una fuente potencial de contaminación, principalmente por los metales pesados y fácilmente lixiviables que pueden contener ( $\mathrm{Pb}, \mathrm{Zn}$ y $\mathrm{Cd}$ ). Sin embargo, también despiertan un gran interés en el desarrollo de técnicas para su tratamiento, debido al valor agregado que les da su alto contenido de zinc. A pesar de que se han implementado algunos métodos para su reciclaje, estos se caracterizan por demandar gran consumo de energía y operar a condiciones extremas. Por tanto, en este trabajo se presenta una alternativa con enfoque técnico para reciclar los EAFD de manera segura, mediante la extracción selectiva de zinc por vía hidrometalúrgica, permitiendo la obtención de un licor rico en zinc para su posterior recuperación y futura aplicación y un sólido detoxificado rico en hierro, para su recirculación en procesos siderúrgicos.

Métodología: Los experimentos se realizaron a través de lixiviaciones por agitación empleando soluciones de citrato de sodio y ácido nítrico a concentración y agitación moderadas, temperatura y presión ambiente. Algunas de las variables de estudio fueron modificadas a fin de establecer las mejores condiciones para el proceso.

Resultados: Luego de realizar una correlación de resultados y análisis de variables, se determinó que es posible alcanzar extracciones por encima del $50 \%$ de Zn y por debajo del $3 \%$ de Fe con el citrato. Además, que una concentración de 0,5 M y proporción S/L de $25 \mathrm{~g} / 11$ son algunas de las condiciones más favorables para el proceso de extracción.

Conclusiones: Es posible un aprovechamiento del residuo EAFD mediante ruta hidrometalúrgica, empleando un agente orgánico como solución lixiviante. A diferencia del tratamiento con ácido nítrico, la lixiviación con citrato permite extraer selectivamente el metal de interés a condiciones de trabajo moderadas.

Financiamiento: Vicerrectoría de Investigación y Extensión - Universidad Pedagógica y Tecnológica de Colombia (VIEUPTC).

Palabras clave: citrato, EAFD, lixiviación, ácido nítrico, zinc.

\footnotetext{
${ }^{1}$ Ingeniera metalúrgica, magíster en Metalurgia y Ciencia de los Materiales. Estudiante Universidad Pedagógica y Tecnológica de Colombia. Tunja, Colombia. Email: angelajohana.borda@uptc.edu.co

${ }^{2}$ Ingeniero metalúrgico. Estudiante Universidad Pedagógica y Tecnológica de Colombia. Tunja, Colombia. Email: fabianstiven.lopez@uptc.edu.co

${ }^{3}$ Ingeniero metalúrgico, magíster en Metalurgia y Ciencia de los Materiales, doctor en Ingeniería Química. Docente de la Universidad Pedagógica y Tecnológica de Colombia. Tunja, Colombia. Email: robinson.torres@uptc.edu.co

${ }^{4}$ Ingeniero metalúrgico, magíster en Metalurgia. Docente de la Universidad Pedagógica y Tecnológica de Colombia. Tunja, Colombia. Email: mario.parra@uptc.edu.co
} 
Reciclaje de EAFD a través de la lixiviación selectiva de zinc con soluciones de citrato: comparación con un ácido inorgánico

Borda ., J. López ., F. Torres., R. y Parra ., M.

\begin{abstract}
Context: Electric arc furnace dust (EAFD) is a potential source of contamination, mainly due to heavy and easily leachable metals that it can contain $(\mathrm{Pb}, \mathrm{Zn}$, and $\mathrm{Cd})$. However, EAFD also arouses great interest in the development of techniques for its treatment due to the added value that its high zinc content gives it. Although some recycling methods have been implemented, they are characterized by demanding high energy consumption and operating in extreme conditions. Therefore in this work, an alternative with a technical approach is presented to safely recycle EAFD through the selective extraction of zinc by hydrometallurgy obtaining, a liquor rich in zinc for its subsequent recovery and future application and a detoxified solid rich in iron for its recirculation in steel processes.

Methodology: The experiments were carried out through agitation leaching using sodium citrate and nitric acid solutions. They were worked at moderate concentrations and stirring speeds and ambient pressure and temperature. Some of the study variables were modified to establish the best conditions for the process.

Results: After making a correlation about the obtained results and the analysis of the variables, it was determined that it is possible to achieve extractions above $50 \%$ of $\mathrm{Zn}$ and below $3 \%$ of Fe with citrate solutions. Furthermore, a concentration of $0.5 \mathrm{M}$ and an S: $\mathrm{L}$ ratio of $25 \mathrm{~g} / 1 \mathrm{~L}$ are some of the most favorable conditions for the extraction process.

Conclusions: It is possible to properly treat the EAFD residue by a hydrometallurgical route using an organic agent as a leaching solution. Unlike nitric acid treatment, citrate leaching allows the metal of interest to be selectively extracted under moderate working conditions.
\end{abstract}

Financing: Vicerrectoría de Investigación y Extensión - Universidad Pedagógica y Tecnológica de Colombia (VIE-UPTC). Keywords: citrate, EAFD, leaching, nitric acid, zinc.

\title{
Tabla de Contenidos
}

Página

Introducción

Metodología

Caracterización de la muestra . . . . . . . . . . . . . . . . . . . . . 135

Soluciones de lixiviación . . . . . . . . . . . . . . . . . . . . . . . . 135

$\begin{array}{ll}\text { Resultados } & 136\end{array}$

Caracterización de la muestra . . . . . . . . . . . . . . . . . . . . . . . . 136

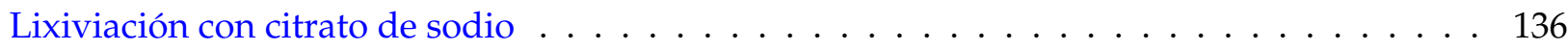

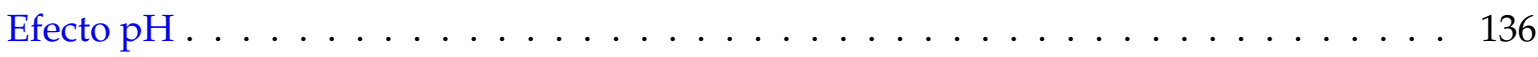

Efecto de la proporción sólido/líquido $(\mathrm{s} / \mathrm{l}) \ldots \ldots \ldots \ldots$. . . . . . . . . . . . . . . . . . . . . . . . .

Efecto de la adición de hidracina . . . . . . . . . . . . . . . . . . . . 141

Lixiviación con citrato en comparación con $\mathrm{HNO}_{3} \ldots \ldots \ldots \ldots \ldots \ldots$

Efecto de la concentración de reactivos . . . . . . . . . . . . . . . . . . . . . . . . . . . . . . . . 14

Efecto de la velocidad de agitación . . . . . . . . . . . . . . . . . . . . . . 144 


\section{Introducción}

Dentro de los principales problemas que presenta la industria siderúrgica en todo el mundo, se encuentran la disposición y tratamiento de los desechos que esta produce. El horno de arco eléctrico, por ejemplo, genera los polvos de acería (electric arc furnace dust, EAFD) como resultado de sus procesos de fusión y afino. Estos se caracterizan por tener una composición química variable y heterogénea que depende directamente de las características de la carga metálica utilizada y las condiciones del proceso (Siame et al., 2019). No obstante, son comunes metales pesados susceptibles de ser lixiviados como plomo y cadmio en su composición, por lo que se les ha llegado a catalogar como un tipo de residuo peligroso (Hagni et al., 1991, Castells, 2000, Bruckard et al., 2005).

Se estima que a nivel mundial la industria siderúrgica genera un poco más de 5 millones o 7 millones de toneladas de EAFD al año (Machado et al., 2006). La preocupación con respecto a su generación radica en que en muchos casos las industrias optan por depositarlos en patios de acopio o vertederos expuestos a la atmosfera, a la espera de la implementación de un enfoque técnico para tratarlos o reciclarlos de manera segura (Siame et al., 2019). La composición misma del material permite una recirculación en el interior de la planta, puesto que su contenido de hierro es considerable. Sin embargo, no se han trazado estrategias novedosas para recuperar el zinc.

Recientemente se han enfocado esfuerzos en recuperar metales desde distintos tipos de residuos (electrónicos, industriales, etc.), para minimizar el impacto ambiental generado durante la obtención de materias primas en procesos metalúrgicos tradicionales (Torres y Lapidus, 2016, Hong et al., 2015, Klemeš et al., 2019); además, resulta ser una alternativa tecnológicamente interesante, debido al consumo de las reservas minerales. En el caso particular del zinc en los residuos EAFD se conocen estudios piro- e hidrometalúrgicos. El sistema Waelz (Alemania) se caracteriza por la volatilización de metales como $\mathrm{Zn}, \mathrm{Pb}$ y $\mathrm{Cd}$ a partir de una mezcla de óxidos sólidos, por medio de la reducción con coque en un horno rotatorio (Madias, 2009). Sin embargo, este proceso presenta desventajas como la dificultad en la regulación de la temperatura, la composición de la carga, las pérdidas irrecuperables de hierro al alcanzar el 50 \% de óxidos en la escoria (Doronin y Svyazhin, 2011, Madias, 2009) y la generación significativa de residuos, ya que no pueden recuperarse algunos metales presentes, como el cobre y el níquel, los cuales permanecen en las escorias (Walburga Keglevich de Buzin et al., 2017). El reciclaje directo de EAFD al horno de arco eléctrico causa muchos problemas operativos, como la 
Reciclaje de EAFD a través de la lixiviación selectiva de zinc con soluciones de citrato: comparación con un ácido inorgánico

Borda ., J. López ., F. Torres., R. y Parra ., M.

falla del refractario y el llenado completo de la extracción de gas (Walburga Keglevich de Buzin et al., 2017).

A pesar de la funcionalidad del proceso de fusión, es evidente el considerable gasto energético por cada kilogramo del metal no ferroso recuperado. La incorporación de los polvos de acería en otros procesos o productos también se ha reportado como método de tratamiento del residuo; los EAFD se han empleado como materia prima secundaria en la producción de morteros autocompactantes (Lozano et al., 2019a, Lozano et al., 2019b) y en las pastas de cemento Portland I modificadas con Pozzolan (De Vargas et al., 2006), entre otros; sin embargo, a pesar de su uso como forma de tratamiento de residuos industriales, trabajos previos han demostrado que la incorporación de materiales reciclados a los compuestos cementicios conduce a un deterioro en su comportamiento físico y mecánico resistente (Xargay et al., 2019,Palacio-León et al., 2017, Robayo-Salazar et al., 2015), requiriendo una vigilancia constante tanto en el proceso como en los productos fabricados.

En las rutas hidrometalúrgicas existen estudios donde se emplean ácidos inorgánicos convencionales y otros alternativos (ácido acético y tartárico) (De la Torre et al., 2013). Se han reportado, por ejemplo, recuperaciones de zinc superiores al $70 \%$ empleando soluciones $6 \mathrm{M}$ de $\mathrm{NaOH}$ a 90 ${ }^{\circ} \mathrm{C}$ (Dutra et al., 2006), mientras que $\mathrm{el}_{2} \mathrm{SO}_{4}$ ha alcanzado extracciones máximas del $80 \%$ a $1,5 \mathrm{M} \mathrm{y}$ $60{ }^{\circ} \mathrm{C}$ (Oustadakis et al., 2010). La desventaja de estos procesos consiste en las extremas condiciones de operación, además, de que algunos reactivos utilizados se clasifican como tóxicos y corrosivos; sin embargo, el principal inconveniente es la nula selectividad del proceso. Como se indicó anteriormente, los metales contenidos dependen del tipo de carga que se emplee en el horno. No obstante, al tratarse de procesos siderúrgicos, el contenido de hierro en la mayoría de casos es elevado. De esta forma, si durante el proceso de lixiviación no se cuenta con algún tipo de selectividad, se tendrán serias dificultades durante las etapas posteriores de electrorrecuperación de cada metal (Torres y Lapidus, 2017).

En estudios anteriores se ha demostrado que las soluciones basadas en citrato de sodio $\left(\mathrm{Na}_{3} \mathrm{C}_{6} \mathrm{H}_{5}\right.$ $\mathrm{O}_{7 \cdot 2} \mathrm{H}_{2} \mathrm{O}$ ) tienen la capacidad de adquirir características de selectividad para determinados metales dependiendo del pH (Torres y Lapidus, 2016). Adicionalmente, este tipo de soluciones lixiviantes poseen la capacidad de ser reutilizadas en varios ciclos de lixiviación/electrorrecuperación, sin perjuicio considerable en su capacidad de disolución de metales (Torres y Lapidus, 2017). De esta forma, el presente estudio tiene como objetivo proponer una ruta selectiva de lixiviación para recuperar el zinc contenido en los EAFD, empleando soluciones reutilizables de citrato que minimicen el potencial impacto ambiental de estos residuos. Se varió la concentración de reactivo y la velocidad de agitación para analizar su comportamiento en el sistema. Los resultados se confrontaron con los encontrados utilizando soluciones de $\mathrm{HNO}_{3}$ bajo las mismas condiciones, para establecer comparación entre el agente orgánico propuesto y un inorgánico convencional. 


\section{Metodología}

\section{Caracterización de la muestra}

El estudio se realizó utilizando EAFD recolectado de una planta colombiana con un horno eléctrico que procesa acero a partir de chatarra. La concentración y el análisis elemental se determinaron mediante digestión química con agua regia $\left(\mathrm{HCl}: \mathrm{HNO}_{3}, 3: 1\right)$.

Los compuestos que se ajustan al EAFD se determinaron mediante la técnica de difracción de rayos X (DRX) en el equipo Panalytical X'pert Pro, que utiliza geometría Bragg-Brentano con tubo de cobalto; las muestras se analizaron con la base de datos ICDD ${ }^{\circledR}$ (International Center for Diffraction Data).

La clasificación granulométrica EAFD se determinó por vía seca utilizando tamices de la serie ASTM-E-11. Las características magnéticas de la muestra generaron aglomeraciones durante el proceso. Para reducir el riesgo de adhesión entre partículas, fueron necesarias pruebas de tamizaje en húmedo. De esta forma, se obtuvo el análisis de tamaño de grano más preciso.

\section{Soluciones de lixiviación}

Se prepararon soluciones de lixiviación usando agua desionizada y citrato de sodio de grado analítico. Las concentraciones molares fueron específicas para cada experimento. El pH se ajustó con una solución diluida de $\mathrm{HNO}_{3}$ (J.T. Baker®). Teórica y experimentalmente se verificó que la química del proceso no se viera afectada por el ajuste (datos no incluidos). El proceso de lixiviación se llevó a cabo a temperaturas entre $15-17^{\circ} \mathrm{C}$. Se utilizaron diferentes proporciones sólido/líquido. Se tomaron alícuotas en momentos específicos para evaluar el comportamiento y la evolución de la lixiviación. En algunos de los experimentos, se añadió hidracina durante cada hora del proceso, para verificar su efecto sobre la velocidad de reacción. Se emplearon agitadores mecánicos tipo paleta, sin bafles (Model 50006-03 - COLE-PARMER). El potencial de las soluciones fue monitorizado empleando un electrodo de $\mathrm{Ag} / \mathrm{AgCl}$ saturado (Oakton pH ORP 700 Benchtop Meter); posteriormente, los valores fueron ajustados al electrodo estándar de hidrógeno (SHE). Todos los diagramas de áreas predominantes se realizaron utilizando el software MEDUSA® (Eriksson, 1979, Puigdomenech, 2004). Los datos termodinámicos correspondientes se contrastaron con la base de datos NIST 46 (NIST, 2004).

Los contenidos metálicos de todas las muestras se cuantificaron con el espectrómetro de emisión atómica de plasma por microondas (Agilent 4210 MP-AES), utilizando estándares y parámetros analíticos establecidos por el fabricante. 


\section{Resultados}

\section{Caracterización de la muestra}

El EAFD está compuesto principalmente de hierro y zinc (tabla 1). El alto contenido de zinc resulta atractivo, considerando que el valor asociado a la recuperación de este metal contribuye significativamente a la viabilidad económica del proceso (Walburga Keglevich de Buzin et al., 2017).

Tabla 1. Contenido de metales pesados de EAFD

\begin{tabular}{ccccc}
\hline Elemento & $\mathbf{C u}$ & $\mathbf{P b}$ & $\mathrm{Fe}$ & $\mathrm{Zn}$ \\
\hline Contenido (\%) & 0,219 & 1,660 & 31,610 & 24,682 \\
\hline
\end{tabular}

Fuente: elaboración propia.

El patrón de difracción de rayos $\mathrm{X}$ se muestra en la figura 1. Las fases predominantes de EAFD son óxidos mixtos con estructura de espinela normal $\left(\mathrm{ZnFe}_{2} \mathrm{O}_{4}\right.$ y $\left.\mathrm{Fe}_{3} \mathrm{O}_{4}\right)$ y óxidos de hierro y zinc $\left(\mathrm{Fe}_{2} \mathrm{O}_{3}, \mathrm{ZnO}\right)$. También se encuentran presentes óxidos de plomo, cobre y silicio, pero en menores cantidades $\left(\mathrm{PbO}, \mathrm{Cu}_{2} \mathrm{O}\right.$ y $\left.\mathrm{SiO}_{2}\right)$.

La composición de esta muestra EAFD concuerda con los hallazgos de otros autores (Hagni et al., 1991, Simonyan et al., 2019).

El estudio de clasificación granulométrica evidenció que la sustancia tiene un tamaño de partícula distribuido de manera homogénea (tabla 2); es decir, la granulometría del EAFD no tiene efecto sobre la composición del lote de muestra; por consiguiente, no fue necesario seleccionar tamaños de partícula específicos para el desarrollo experimental.

Tabla 2. Determinación granulométrica de EAFD

\begin{tabular}{ccccccccc}
\hline Abertura $(\mu \mathbf{m})$ & $\mathbf{4 2 5}$ & $\mathbf{2 5 0}$ & $\mathbf{1 5 0}$ & $\mathbf{1 0 6}$ & $\mathbf{7 5}$ & $\mathbf{6 3}$ & Fondo & Total \\
\hline Peso retenido (\%) & 15,92 & 12,29 & 17,22 & 12,39 & 14,77 & 10,84 & 16,57 & 100 \\
\hline
\end{tabular}

Fuente: elaboración propia.

\section{Lixiviación con citrato de sodio}

\section{Efecto $p H$}

Para determinar el área de estabilidad de los metales determinados en la composición del residuo en soluciones de citrato, fue necesario hacer un análisis termodinámico. La información proporcio- 


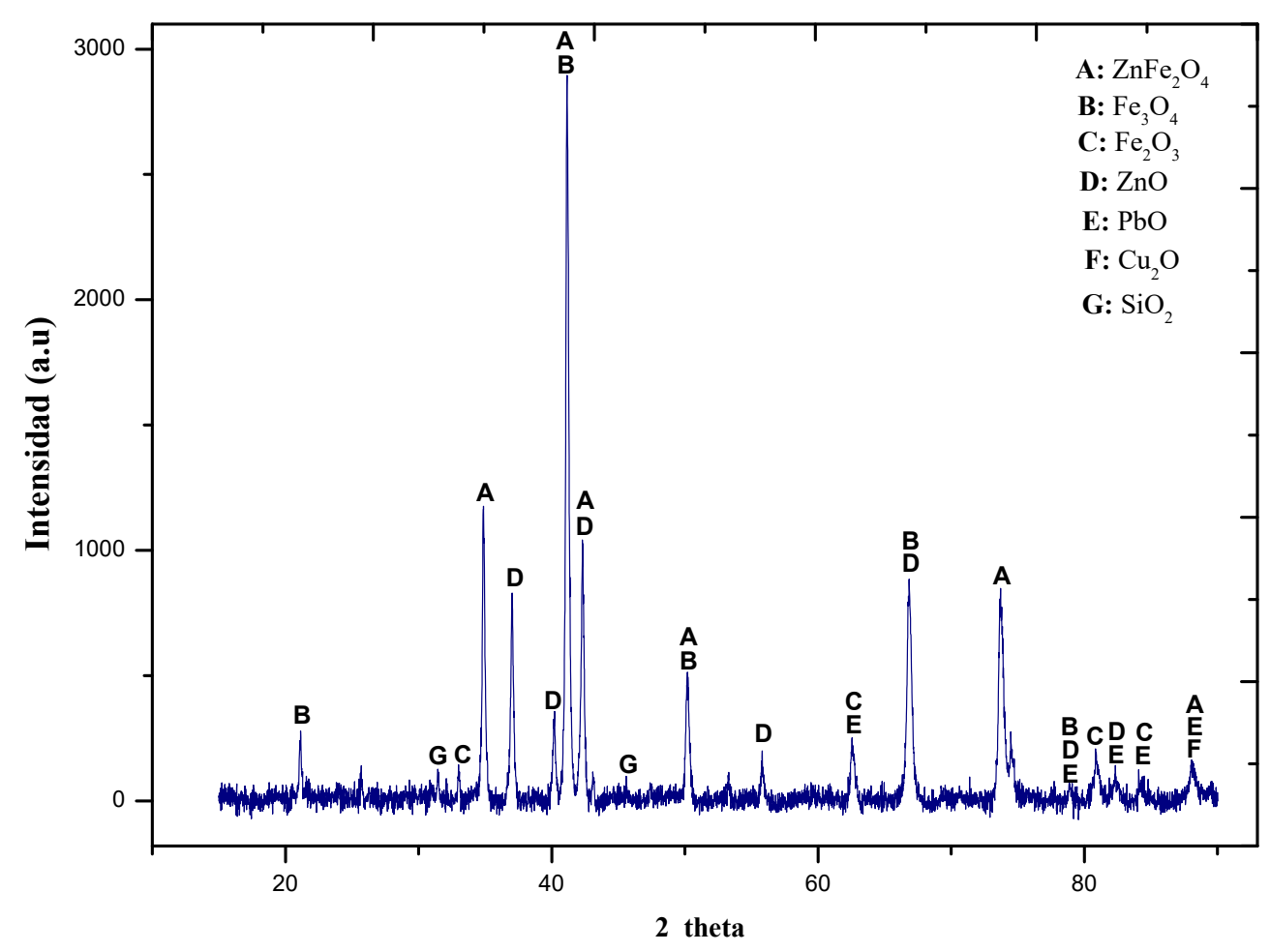

Figura 1. Difractograma de EAFD

Fuente: elaboración propia.

nada por los diagramas de predominio (figura 2) permitió ubicar las condiciones termodinámicas favorables para la lixiviación de los metales de estudio.

El citrato forma complejos solubles con los iones metálicos según el pH y los potenciales de solución. El potencial del sistema M-Cit fue de 370 a $490 \mathrm{mV}$. Sin embargo, el análisis termodinámico de metales muestra que la formación de complejos está limitada por el pH y no por el potencial. Por tanto, el potencial de solución no se discute en este estudio. El área de estabilidad del zinc está en el rango de $\mathrm{pH}$ de 1 a 10,5; sin embargo, los otros metales también tienen una amplia gama de formación de especies solubles. Para verificar el efecto de las soluciones en condiciones básicas, neutras y ácidas, se realizaron experimentos variando el pH en 3,0; 6,0 y 9,0 (figura 3).

Las pruebas de lixiviación fueron favorables a $\mathrm{pH} 3$ y 6 , aunque la superioridad del $\mathrm{pH}$ ácido fue del $12 \%$. Mientras tanto, en condiciones alcalinas, el citrato solo logró disolver el $10 \%$ del zinc. La formación de complejos a partir del ligando orgánico $\mathrm{Cit}^{3-}$ y el catión divalente $\mathrm{Zn}^{+2}$, depende del $\mathrm{pH}$. 

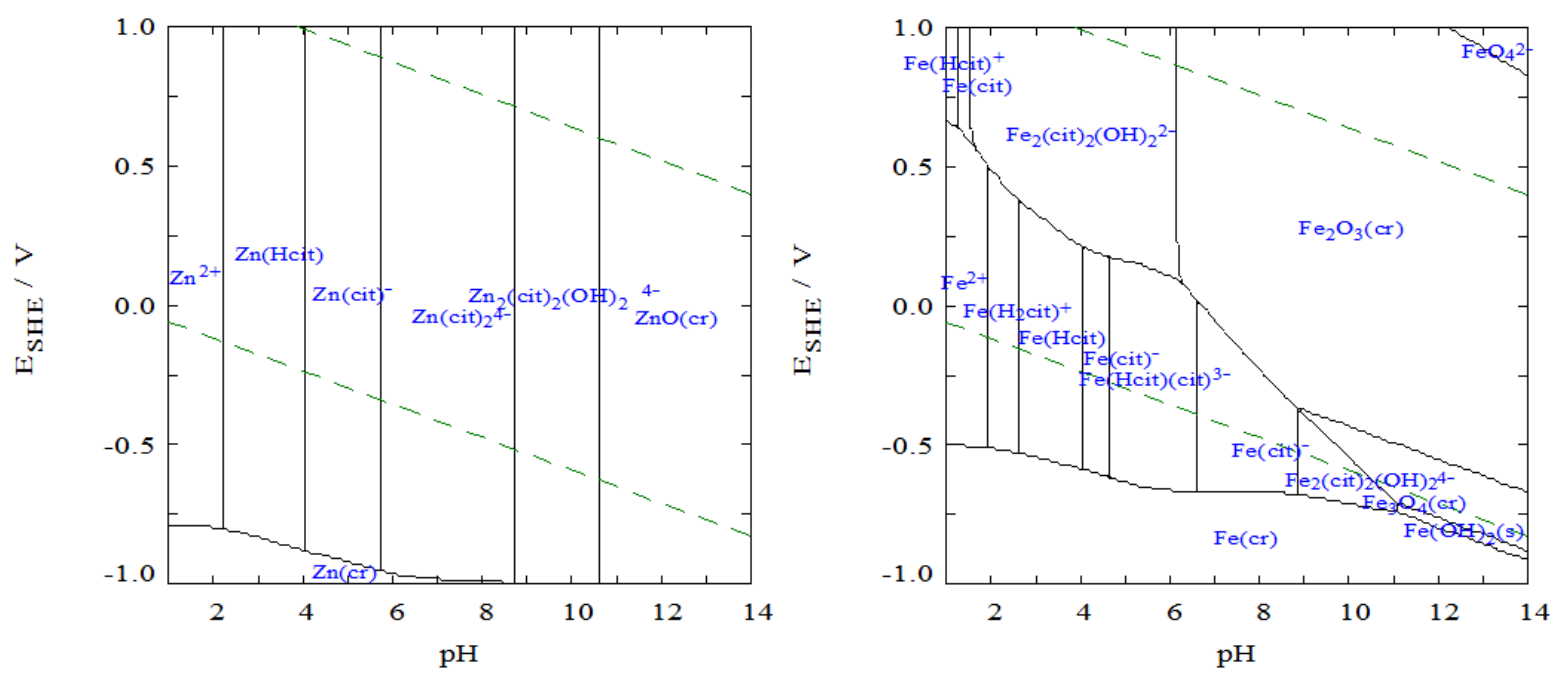

(a)

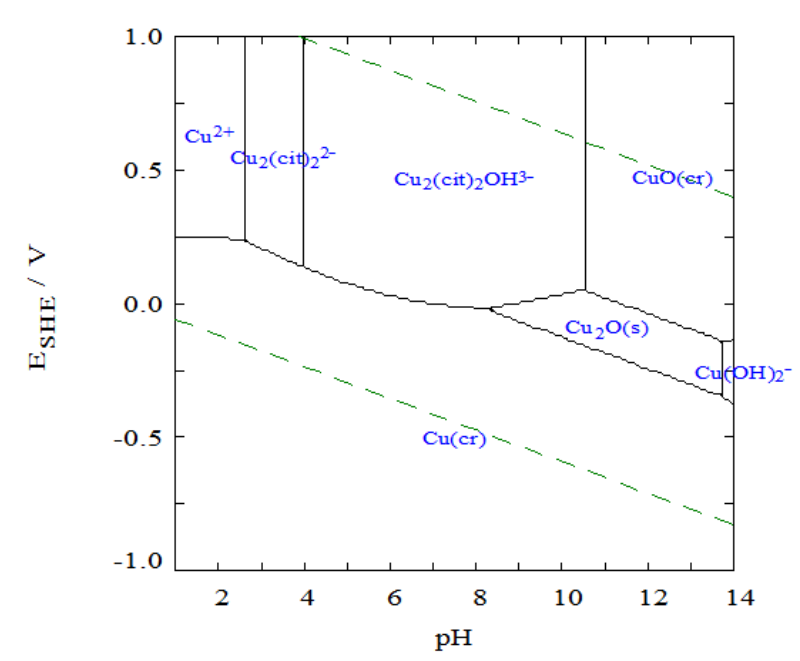

(c) (b)

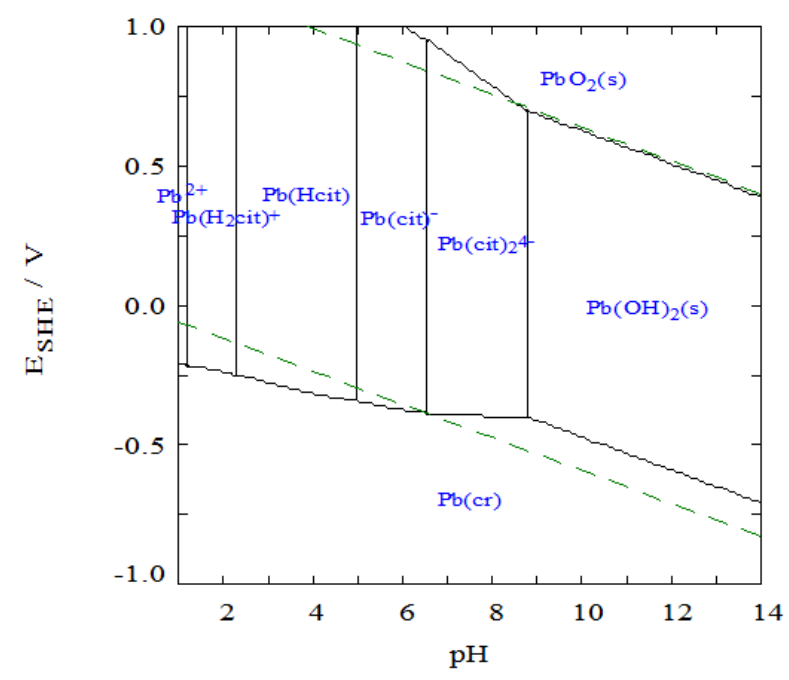

(d)

Figura 2. Diagramas de predominio para zinc (a), hierro (b), cobre (c) y plomo (d) en presencia de citrato 0,5 M. Diseñado con el software MEDUSA

Fuente: elaboración propia.

Según los datos termodinámicos, el sistema se ve favorecido por la presencia del ion hidronio, alcanzando el estado energético más estable a pH 3 (tabla 3; ecuaciones (1), (5) y (9)).

Por tanto, la disolución de zinc en estado oxidado se beneficia en medios ácidos. Los resultados son prometedores en cuanto a la baja disolución de hierro que alcanza el citrato $(<3 \%)$ al $3 \mathrm{pH}$ trabajado. El citrato muestra su capacidad selectiva para no disolver óxidos de hierro (figura 3). Su energía libre denota que son inestables y menos espontáneos que los formados por zinc (tabla 3, ecuaciones 

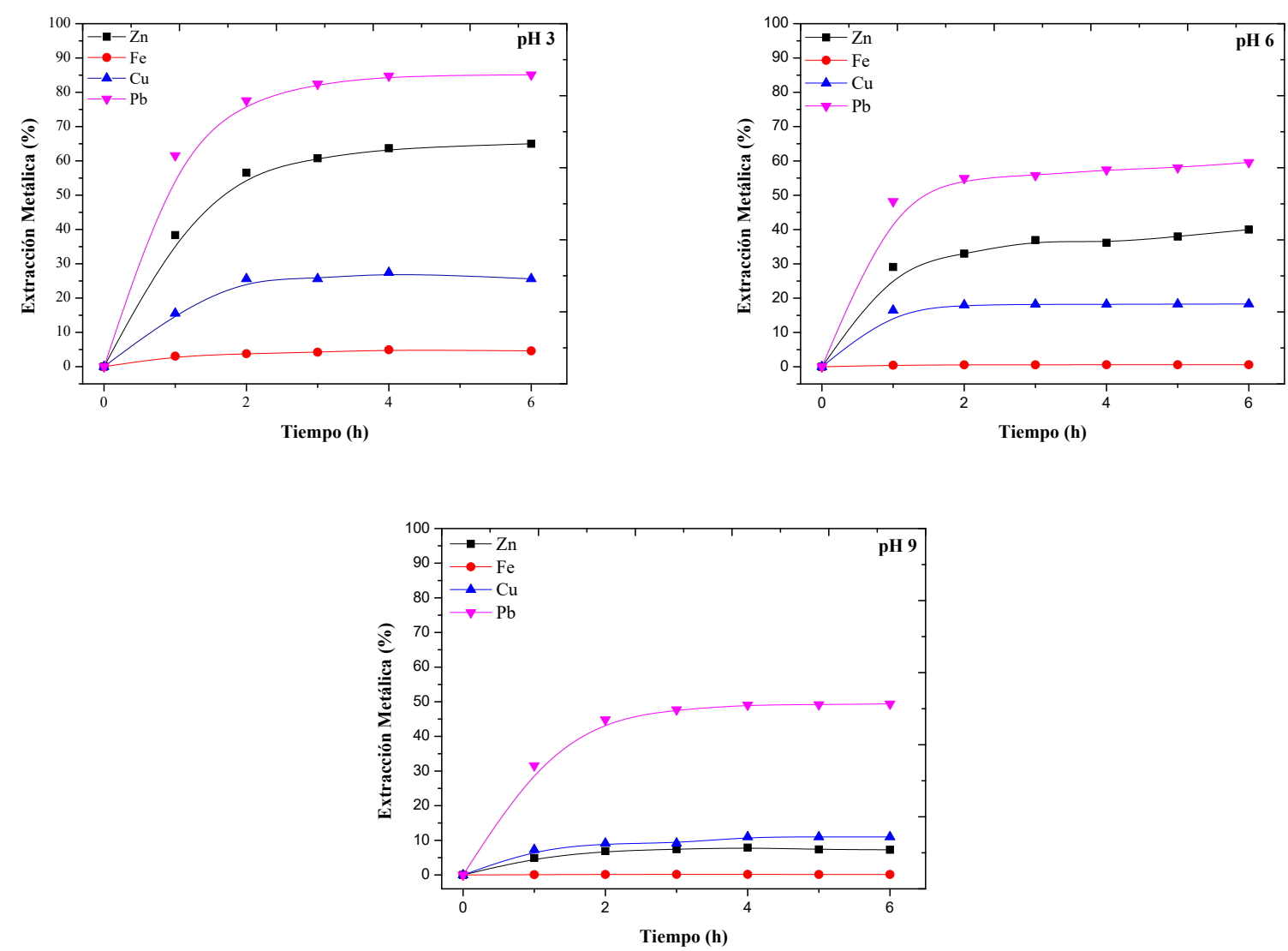

Figura 3. Efecto del pH sobre la extracción de metales contenidos en EAFD. Condiciones: citrato de sodio 0,5 M а pH 3, pH 6 у pH 9; 500 r. p. m.; 25 g EAFD/1

Fuente: elaboración propia.

(4), (4) y (12)). Adicionalmente, en el estudio de difracción de rayos X (figura 1) se encontró que el hierro corresponde principalmente a una especie cristalina bastante estable (Carriazo Baños et al., 2017) y poco soluble $\left(\mathrm{Fe}_{3} \mathrm{O}_{H N O}\right)$ que no se degrada en la lixiviación.

Termodinámicamente, tanto el cobre como el plomo tienen condiciones de $\mathrm{pH}$ favorables para la disolución (figura 2c-d). Pero, debido a su baja concentración en el EAFD y su modesta extracción (figura 2), el cobre no se tuvo en cuenta en el presente estudio. Para asegurar la lixiviación selectiva de zinc, se añadió el anión $\mathrm{SO}_{2}^{-4}$ como ligando. Estas moléculas forman una especie insoluble con el plomo e inhiben su disolución. A pesar de variar la concentración de ligando, los resultados no fueron los esperados (datos no incluidos), ya que la disolución de $\mathrm{Pb}$ en citrato tuvo lugar en el proceso. Una posible solución a esto es realizar posteriores tratamientos de cementación o precipitación del plomo; sin embargo, estas pruebas están fuera del alcance de este estudio. 
Reciclaje de EAFD a través de la lixiviación selectiva de zinc con soluciones de citrato: comparación con un ácido inorgánico

Borda ., J. López ., F. Torres., R. y Parra ., M.

Tabla 3. Datos termoquímicos para el producto de reacción de la lixiviación de $\mathrm{Zn}, \mathrm{Cu}, \mathrm{Pb}$ y Fe

\begin{tabular}{|c|c|c|c|}
\hline pH & Reacción & & Delta G \\
\hline \multirow{4}{*}{3} & $Z n^{2+}+H^{+}+C i t^{3-}=Z n(H C i t)$ & (1) & $-13991,2$ \\
\hline & $2 C u^{2+}+2 C i t^{3-}=C u_{2}(C i t)_{2}^{2-}$ & (2) & $-23550,5$ \\
\hline & $\mathrm{Pb}^{2+}+\mathrm{H}^{+}+\mathrm{Cit}^{3-}=\mathrm{Pb}(\mathrm{HCit})$ & (3) & $-15286,7$ \\
\hline & $2 \mathrm{Fe}^{3+}+2 \mathrm{Cit}^{3-}=2 \mathrm{H}^{+}+\mathrm{Fe}_{2}(\mathrm{Cit})^{2}(\mathrm{OH} 2)^{2-}$ & (4) & 2045,5 \\
\hline \multirow{4}{*}{6} & $Z n^{2+}+2 C i t^{3-}=Z n(C i t)^{4-}$ & (5) & $-10186,6$ \\
\hline & $2 \mathrm{Cu}^{2+}+2 \mathrm{Cit}^{3-}=\mathrm{H}^{+}+\mathrm{Cu}_{2}(\mathrm{Cit})_{2} \mathrm{OH}^{3-}$ & (6) & $-18136,76$ \\
\hline & $\mathrm{Pb}^{2+}+\mathrm{Cit^{3- }}=\mathrm{Pb}(\mathrm{Cit})^{-}$ & (7) & $-8550,2$ \\
\hline & $2 \mathrm{Fe}^{3+}+2 \mathrm{Cit}^{3-}=2 \mathrm{H}^{+}+\mathrm{Fe}_{2}(\mathrm{Cit})_{2}\left(\mathrm{OH}_{2}\right)^{2-}$ & (8) & 2045,5 \\
\hline \multirow{4}{*}{9} & $2 \mathrm{Zn}^{2+}+2 \mathrm{Cit}^{3-}=2 \mathrm{H}^{+}+\mathrm{Zn}_{2}(\mathrm{Cit})_{2}(\mathrm{OH})_{2}^{4-}$ & (9) & 2740,97 \\
\hline & $2 \mathrm{Cu}^{2+}+2 \mathrm{Cit}^{3-}=\mathrm{H}^{+}+\mathrm{Cu}_{2}(\mathrm{Cit})_{2} \mathrm{OH}^{3-}$ & (10) & $-18136,76$ \\
\hline & $P b^{2+}=2 H^{+}+P b(O H)^{2}(s)$ & (11) & 11113,9 \\
\hline & $2 \mathrm{Fe}^{2+}=6 \mathrm{H}^{+}+2 e^{-}+\mathrm{Fe}_{2} \mathrm{O}_{3}$ & (12) & 36066,2 \\
\hline
\end{tabular}

Fuente: (Puigdomenech, 2004) y (NIST, 2004).

\section{Efecto de la proporción sólido/líquido (s/l)}

El efecto de la variación en la proporción s/1 sobre la disolución de zinc en solución de citrato se muestra en la figura 4. Como se esperaba, la eficiencia de lixiviación de zinc disminuyó con la relación más alta probada (C). La saturación de la solución o la limitación estequiométrica del citrato inhibió la disolución completa de los metales contenidos en la muestra.

La proporción de 12,5 g/l (A) muestra disoluciones favorables; sin embargo, la mayor extracción se alcanza con la proporción de $25 \mathrm{~g} / \mathrm{l}$ (B). La diferencia entre estos ensayos no es significativa ni proporcional; además, a nivel industrial, no resulta eficiente procesar pocas cantidades de material en las 


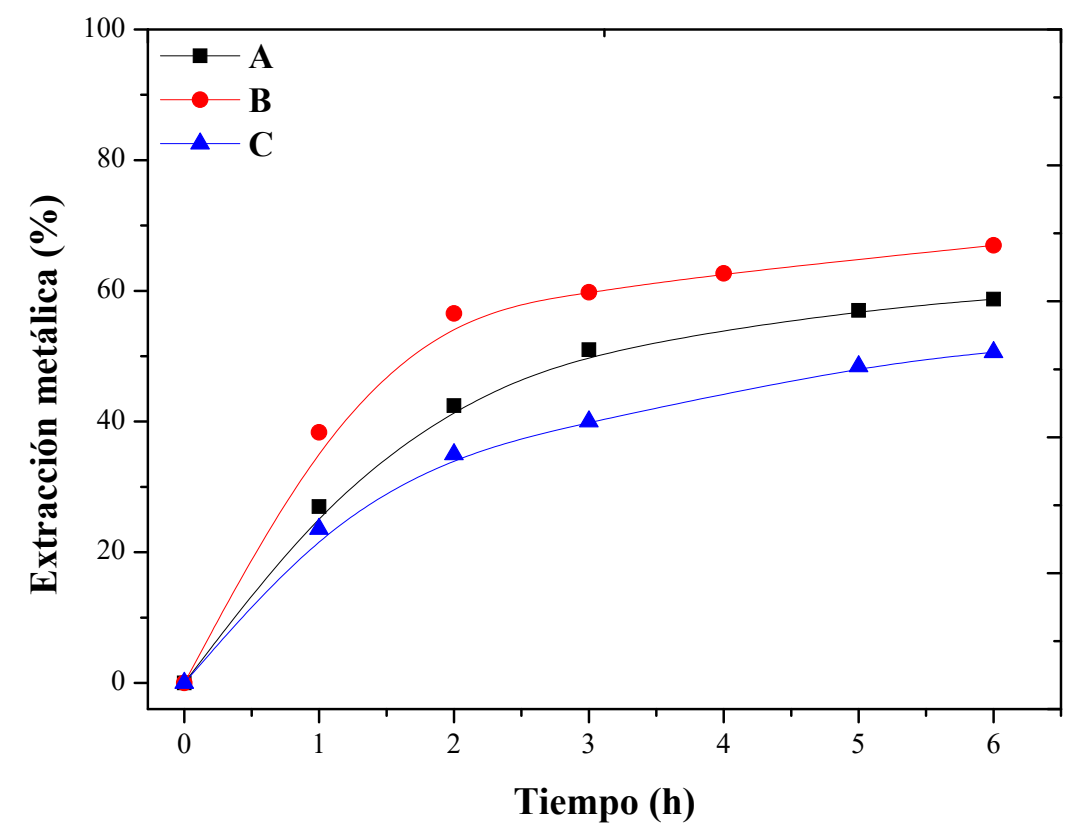

Figura 4. Influencia de la relación S/L en el comportamiento de lixiviación del zinc de EAFD. Condiciones: (A) 12,5, (B) 25 y (C) 50 g/L. Citrato 0,5 M, pH 3; 500 r. p. m.

Fuente: elaboración propia.

operaciones metalúrgicas, debido a que la cantidad de reactivo empleado tiende a desaprovecharse, por consiguiente, la relación s/1 más adecuada para el tratamiento de EAFD es de $25 \mathrm{~g} / 1$.

\section{Efecto de la adición de hidracina}

Las pruebas preliminares indicaron que la extracción máxima de zinc es aproximadamente del 60 \% en la tercera hora de lixiviación. Pasado este tiempo, la extracción es insignificante. En la mayoría de los procesos hidrometalúrgicos se requieren oxidantes fuertes para incrementar las extracciones (Córdoba et al., 2008), sin embargo, entre los componentes del EAFD (figura 1), solo el $\mathrm{Fe}_{2} \mathrm{O}_{3}$ podría oxidarse. Por tanto, se puede anticipar que la adición de estos agentes tiene poco efecto sobre el aumento de la lixiviación de zinc. La zincita $(\mathrm{ZnO})$ y franklinita $\left(\mathrm{ZnFe}_{2} \mathrm{O}_{4}\right)$, son las fases en las que se encuentra contenido el metal no ferroso en el residuo; la lixiviación de $\mathrm{ZnO}$ es posible en medios alcalinos o ácidos (Chairaksa et al., 2016), pero la situación del ZnFe 2 O 4 es diferente. Su estabilidad requiere condiciones termodinámicas más drásticas para su descomposición (Zhang et al., 2011), por ende, se añadió hidracina $\left(\mathrm{N}_{2} \mathrm{H}_{4}\right)$ 0,1 M durante la lixiviación con el objeto de reducir el hierro de la ferrita $\left(\mathrm{Fe}^{+3} \rightarrow \mathrm{Fe}^{+2}\right)$ y así liberar y extraer los iones zinc. Los resultados se muestran en la figura 5 . 


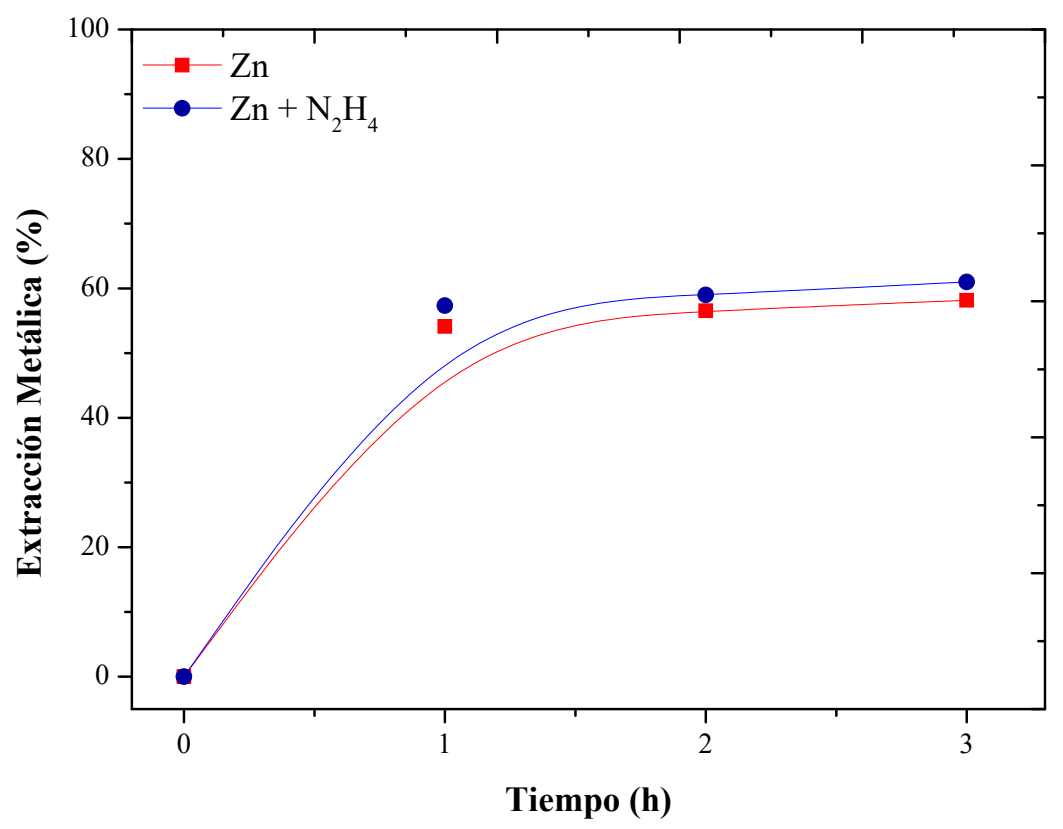

Figura 5. Efecto de la adición de hidracina. Condiciones: Citrato de sodio a pH 3 y 0,5 M; 500 r. p. m.; 25 g $\mathrm{EAFD} / 1$

Fuente: elaboración propia.

La acción del $\mathrm{N}_{2} \mathrm{H}_{4}$ no es suficiente para mejorar el proceso de recuperación. Posiblemente la descomposición de $\mathrm{ZnFe}_{2} \mathrm{O}_{4}$ se centre en procesos pirometalúrgicos (Yang et al., 2011, ChairaksaFujimoto et al., 2016). Por tanto, está claro que independientemente de si se utilizan agentes oxidantes o reductores, la espinela es muy difícil de disolver químicamente en soluciones acuosas para mejorar la extracción de zinc.

Los resultados no muestran la importancia y la necesidad de continuar lixiviando después de la tercera hora.

\section{Lixiviación con citrato en comparación con $\mathrm{HNO}_{3}$}

Los iones de nitrato permiten la formación de especies metálicas con una solubilidad moderada permitiendo un $48 \%$ de extracción de zinc. Sin embargo, los óxidos de hierro también son ligeramente estables en medios de nitrato y alcanzan aproximadamente el $10 \%$ de extracción (figura 6). Este contenido de hierro disuelto requiere un proceso de purificación de licor. La extracción de zinc con citrato fue superior a la obtenida con $\mathrm{HNO}_{3}$. Además, el citrato es más selectivo, ya que logró disolver menores cantidades de hierro. Sin embargo, el comportamiento de las curvas es similar para 


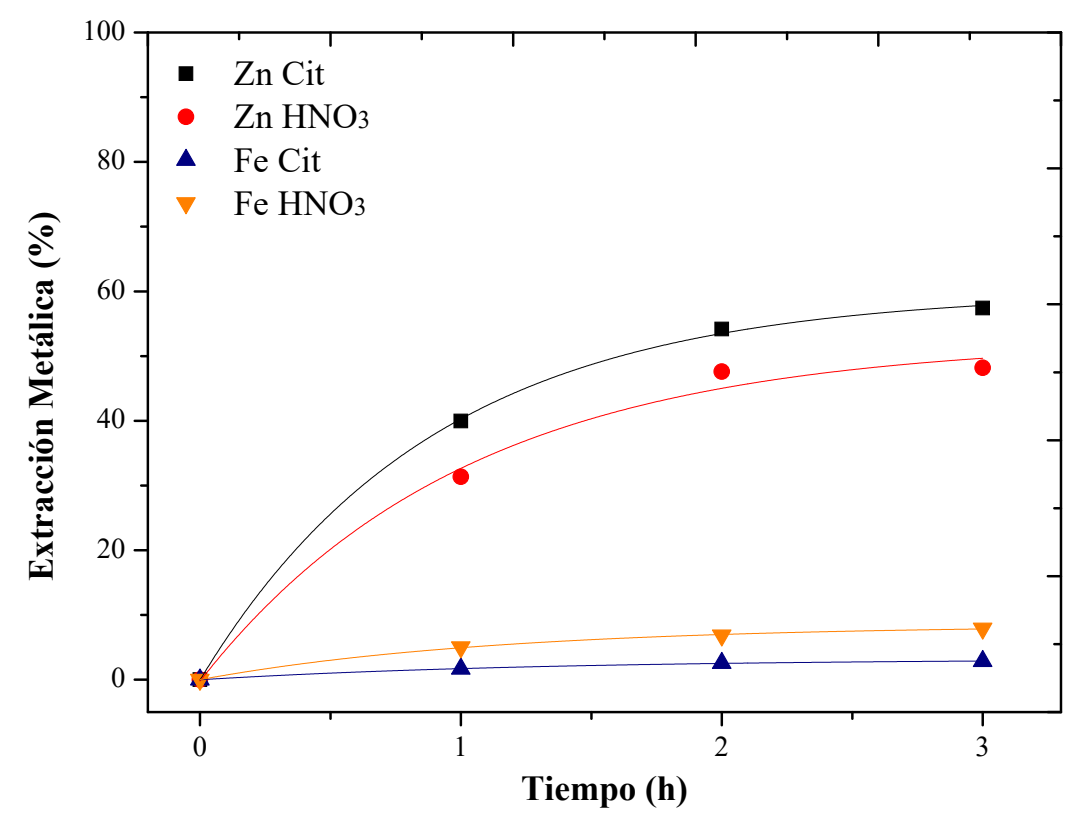

Figura 6. Lixiviación con citrato vs. HNO 3 . Condiciones: citrato y HNO 3 0,5 M; 500 r. p. m.; 25 g EAFD/1

Fuente: elaboración propia.

ambos agentes. Ambos sistemas exhiben una tasa de extracción rápida debido a la alta solubilidad de $\mathrm{ZnO}$ y al pequeño tamaño de las partículas de EAFD (Nagib e Inoue, 2000).

\section{Efecto de la concentración de reactivos}

Se estudió el efecto de la concentración de reactivos sobre la velocidad de reacción de la lixiviación. Se puede ver que la eficiencia de la extracción de zinc aumenta ligeramente al aumentar la concentración de reactivos (figura 7).

Sin embargo, al duplicar la concentración de reactivo, la extracción mejora en un $8 \%$ en el mejor de los casos. Esta falta de proporcionalidad indica que la concentración es aparentemente independiente de la recuperación del metal.

Las reacciones de los sistemas $\mathrm{M}$-Cit y $\mathrm{M}-\mathrm{HNO}_{3}$ muestran el comportamiento descrito de las reacciones de pseudo primer orden: la cinética no revela una velocidad de reacción constante, ni dependencia alguna de rapidez respecto a la concentración de los reactivos (Avery, 1974). En el caso del hierro, la concentración tiene un efecto mayor sobre el $\mathrm{HNO}_{3}$ que sobre el citrato; sin embargo, el incremento en la extracción es solo del $2 \%$. 


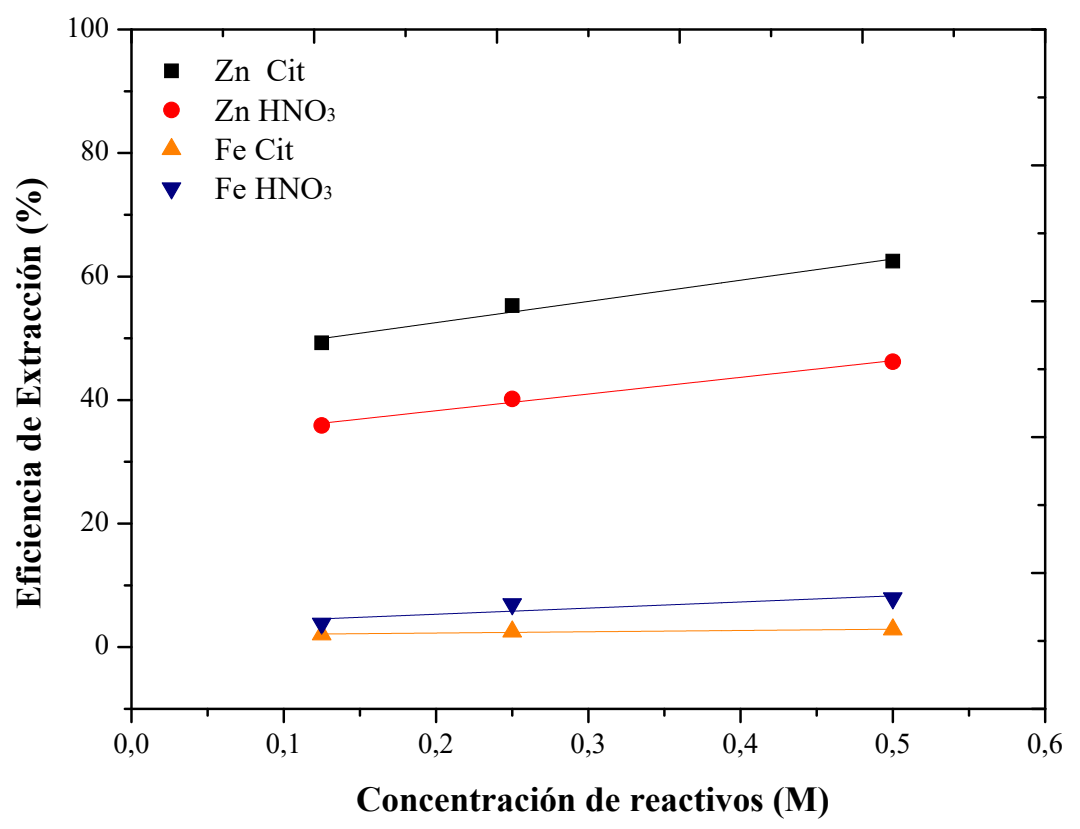

Figura 7. Efecto de la concentración de reactivos sobre la eficiencia de extracción de Zn y Fe. Condiciones: citrato de sodio y $\mathrm{HNO}_{3}$ a 0,125; 0,25 y 0,5 M; 0,5 M; 500 r. p. m.; 25 g EAFD/1

Fuente: elaboración propia.

\section{Efecto de la velocidad de agitación}

Se varió la agitación a 125, 250 y 500 r. p. m., para verificar el efecto del grado de suspensión de los sólidos en la solución. Ambos reactivos tienen un comportamiento similar. Los resultados muestran que la extracción de zinc aumenta ligeramente al aumentar la velocidad de agitación (figura 8). No obstante, el aumento entre estas agitaciones es de menos del $10 \%$ para ambos reactivos; la extracción de hierro, por su parte, no se vio afectada. Al no ser significativos ni proporcionales los aumentos de extracción con la variación de la agitación, se descartan posibles efectos de transferencia de masa. Por tanto, para este estudio la agitación no tiene efecto aparente sobre la cinética de reacción.

Debido al comportamiento de las curvas de extracción, se puede predecir el modelo de control del sistema de lixiviación. La reacción química puede tener lugar rápidamente en la interfaz entre el sólido y el producto de reacción (figura 8), desplazándose hasta el interior de la partícula formando un núcleo de material sin reaccionar (ferritas), dejando en su lugar una capa porosa de sólidos inertes (Levenspiel, 1999). Esto se refleja con el comportamiento de la curva de extracción de zinc, inicialmente, con una elevada pendiente, seguido de un comportamiento asintótico adoptado luego de la primera hora; probablemente la especie $\mathrm{ZnO}$, presenta una lixiviación espontánea, agotándose 


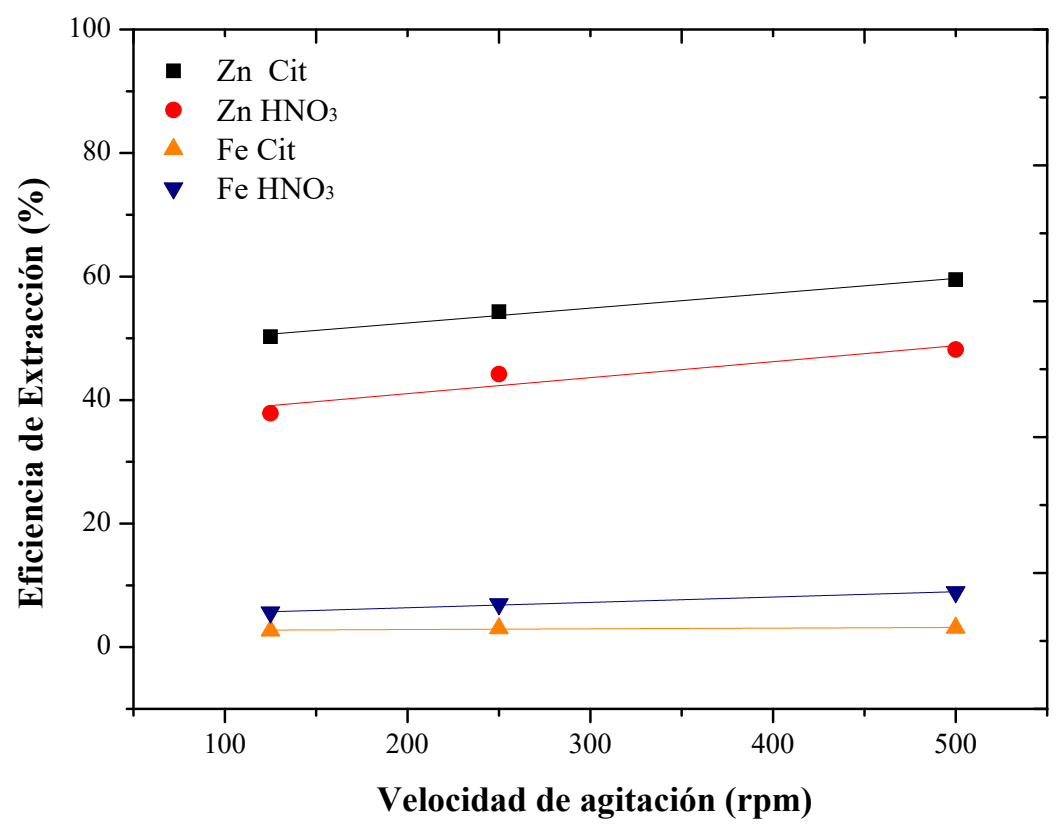

Figura 8. Efecto de la velocidad de agitación sobre la eficiencia de extracción de Zn y Fe. Condiciones: citrato de sodio y $\mathrm{HNO}_{3}$ 0,5 M; 125; 250 y 500 r. p. m.; 25 g EAFD/1

Fuente: elaboración propia.

rápidamente y quedándose sin disolver, la fase de la ferrita $\mathrm{ZnFe}_{2} \mathrm{O}_{4}$.

Los criterios específicos de la cinética del proceso se escapan del alcance de este estudio y están en proceso de publicación por los autores.

\section{Conclusiones}

Las soluciones de citrato proporcionaron una alta selectividad hacia el zinc, ya que solo menos del $3 \%$ de hierro se extrajo de los residuos.

La presencia de franklinita no permite lixiviar el total de zinc presente en EAFD, ya que su estabilidad evita su disolución química en soluciones acuosas.

La adición de ligandos $\mathrm{SO}_{4}^{2-}$ en el proceso no es eficaz para inhibir la disolución del plomo en la solución de citrato; se recomiendan tratamientos poslixiviación para la extracción selectiva de zinc.

La lixiviación con citrato presentó mejores resultados que el $\mathrm{HNO}_{3}$. Extrae más zinc, logra una lixiviación selectiva y resulta más ecológico, al ser orgánico y contar con la posibilidad de reutilizarse en nuevos procesos de lixiviación. 
De acuerdo con el análisis de resultados experimentales en el proceso de lixiviación, se encontró que la mejor extracción de zinc lograda a partir de EAFD se obtiene al realizar el proceso bajo las siguientes condiciones: tres horas de operación; citrato a pH 3 y concentración molar de 0,5; la proporción s/l elegida fue de $25 \mathrm{~g} / 11$ de solución.

Se recomiendan nuevos estudios, mediante los cuales la modificación de las condiciones aquí estudiadas permitan que el proceso encuentre la optimización y el uso adecuado de este tipo de residuos industriales.

\section{Financiamiento}

Vicerrectoría de Investigación y Extensión, Universidad Pedagógica y Tecnológica de Colombia (VIE-UPTC).

\section{Agradecimientos}

Los autores agradecen el apoyo recibido del proyecto VIE-SGI para la realización de esta investigación, así como del Grupo Metalurgia No Ferrosa de la Universidad Pedagógica y Tecnológica de Colombia UPTC.

\section{Referencias}

[Avery, 1974] Avery, H. E. (1974). Basic reaction kinetics and mechanisms. Macmillan Publishers. https://doi.org/10.1007/978-1-349-15520-0个Ver página 143

[Bruckard et al., 2005] Bruckard, W. J., Davey, K. J., Rodopoulos, T., Woodcock, J. T. e Italiano, J. (2005). Water leaching and magnetic separation for decreasing the chloride level and upgrading the zinc content of EAF steelmaking baghouse dusts. International Journal of Mineral Processing, 75(1-2), 1-20. https://doi.org/10.1016/j.minpro.2004.04.007 个Ver página 133

[Carriazo Baños et al., 2017] Carriazo Baños, J. G., Noval Lara, V. E. y Ochoa Puentes, C. (2017). Magnetita (Fe3O4): una estructura inorgánica con múltiples aplicaciones en catálisis heterogénea. Revista Colombiana de Química, 46(1), 42. https://doi.org/10.15446/rev. colomb.quim. v46n1.62831 $\uparrow$ Ver página 139

[Castells, 2000] Castells, X. (2000). Reciclaje de residuos industriales. Ediciones Díaz de Santos S.A. $\uparrow$ Ver página 133 
Reciclaje de EAFD a través de la lixiviación selectiva de zinc con soluciones de citrato: comparación con un ácido inorgánico

Borda ., J. López ., F. Torres., R. y Parra ., M.

[Córdoba et al., 2008] Córdoba, E. M., Muñoz, J. A., Blázquez, M. L., González, F. y Ballester, A. (2008). Leaching of chalcopyrite with ferric ion. Part I: General aspects. Hydrometallurgy, 93(3-4), 81-87.https://doi.org/10.1016/j.hydromet.2008.04.015个Ver página 141

[Chairaksa-Fujimoto et al., 2016] Chairaksa-Fujimoto, R., Maruyama, K., Miki, T. y Nagasaka, T. (2016). The selective alkaline leaching of zinc oxide from Electric Arc Furnace dust pre-treated with calcium oxide. Hydrometallurgy, 159, 120-125. https : / doi . org/10.1016/ j. hydromet . 2015.11.009 Ver página 142

[De la Torre et al., 2013] De la Torre, E., Guevara, A. y Espinoza, C. (2013). Valorización de polvos de acería, mediante recuperación de zinc por lixiviación y electrólisis. Revista Politécnica, 32(1), 51-56. 个Ver página 134

[De Vargas et al., 2006] De Vargas, A. S., Masuero, Â. B., y Vilela, A. C. F. (2006). Investigations on the use of electric-arc furnace dust (EAFD) in Pozzolan-modified Portland cement I (MP) pastes. Cement and Concrete Research, 36(10), 1833-1841. https://doi .org/10.1016/j.cemconres . $2006.06 .003 \uparrow$ Ver página 134

[Doronin y Svyazhin, 2011] Doronin, I. E. y Svyazhin, A. G. (2011). Commercial methods of recycling dust from steelmaking. Metallurgist, 54(9-10), 673-681. https://doi.org/10.1007/ s11015-011-9356-z $\uparrow$ Ver página 133

[Dutra et al., 2006] Dutra, A. J. B., Paiva, P. R. P. y Tavares, L. M. (2006). Alkaline leaching of zinc from electric arc furnace steel dust. Minerals Engineering, 19(5), 478-485. https : / / doi . org/10 . $1016 / j$.mineng.2005.08.013个Ver página 134

[Eriksson, 1979] Eriksson, G. (1979). An algorithm for the computation of aqueous multicomponent, multiphase equilibria. Analytica Chimica Acta, 112,375-383. https: / / doi . org/10.1016/ S0003-2670(01) 85035-2 个Ver página 135

[Hagni et al., 1991] Hagni, A. M., Hagni, R. D. y Demars, C. (1991). Mineralogical characteristics of electric arc furnace dusts. Journal of The Minerals, Metals $\mathcal{E}$ Materials Society, 43(4), 28-30. https: //doi.org/10.1007/BF03220543个Ver página 133, 136

[Hong et al., 2015] Hong, J., Shi, W., Wang, Y., Chen, W. y Li, X. (2015). Life cycle assessment of electronic waste treatment. Waste Management, 38, 357-365. https://doi.org/10.1016/j. wasman.2014.12.022 个Ver página 133

[Klemeš et al., 2019] Klemeš, J. J., Stehlík, P. y Worrell, E. (2010). Waste treatment to improve recycling and minimise environmental impact. Resources, Conservation and Recycling, 54(5), 267- 270. https: //doi.org/10.1016/j.resconrec.2009.11.005 Ter página 133 
Reciclaje de EAFD a través de la lixiviación selectiva de zinc con soluciones de citrato: comparación con un ácido inorgánico

Borda ., J. López ., F. Torres., R. y Parra ., M.

[Levenspiel, 1999] Levenspiel, O. (1999). Chemical reaction engineering. 3a. ed. John Wiley \& Sons, Inc. https://doi.org/10.1021/ie990488g 个Ver página 144

[Lozano et al., 2019a] Lozano, A., Raposeiro, P., Álvarez, De Brito, J. y Jiménez, J. (2019a). Performance and durability properties of self-compacting mortars with electric arc furnace dust as filler. Journal of Cleaner Production, 219, 818-832. https://doi.org/10.1016/j.jclepro.2019.02. $145 \uparrow$ Ver página 134

[Lozano et al., 2019b] Lozano, A., Raposeiro, P., De Brito, J., Fernández, J. M. y Jiménez, J. R. (2019b). Safe use of electric arc furnace dust as secondary raw material in self-compacting mortars production. Journal of Cleaner Production, 211, 1375-1388. 10.1016/j.jclepro.2018.12.002 https : / / doi . org/10.1016/j.jclepro.2018.12.002个Ver página 134

[Machado et al., 2006] Machado, J., Brehm, F., Moraes, C., Santos, C., Vilela, A. y Cunha, J. (2006). Chemical, physical, structural and morphological characterization of the electric arc furnace dust. Journal of Hazardous Materials, 136(3), 953-960. https: / / doi . org/10 .1016/ j . jhazmat. $2006.01 .044 \uparrow$ Ver página 133

[Madias, 2009] Madias, J. (2009). Reciclado de polvos de horno eléctrico. Acero Latinoamericano, 23(513), 38. $\uparrow$ Ver página 133

[Nagib e Inoue, 2000] Nagib, S. e Inoue, K. (2000). Recovery of lead and zinc from fly ash generated from municipal incineration plants by means of acid and/or alkaline leaching. Hydrometallurgy, 56(3), 269-292. https: / / doi .org/10.1016/s0304-386X (00) 00073-6个Ver página 143

[NIST, 2004] NIST. (2004). Critically selected stability constants of metal complexes. NIST Standard Reference Database 46, Version 8.0. $\uparrow$ Ver página 135, 140

[Oustadakis et al., 2010] Oustadakis, P., Tsakiridis, P. E., Katsiapi, A. y Agatzini-Leonardou, S. (2010). Hydrometallurgical process for zinc recovery from electric arc furnace dust (EAFD). Journal of Hazardous Materials, 179(1-3), 1-7. https://doi.org/10.1016/j.jhazmat.2010.01.059个Ver página 134

[Palacio-León et al., 2017] Palacio-León, O., Chávez, A. y Velásquez, Y. (2017). Evaluación y comparación del análisis granulométrico obtenido de agregados naturales y reciclados. Tecnura, 21(53), 96-106. https: / / doi.org/10.14483/22487638.8195 ^ver página 134

[Puigdomenech, 2004] Puigdomenech, I. (2004). HYDRA (Hydrochemical Equilibrium-Constant Database) and MEDUSA (Make Equilibrium Diagrams Using Sophisticated Algorithms) Programs. Royal Institute of Technology. $\uparrow$ Ver página 135, 140

[Robayo-Salazar et al., 2015] Robayo-Salazar, R. A., Mattey-Centeno, P. E., Silva-Urrego, Y. F., BurgosGalindo, D. M. y Arjona, S. (2015). Los residuos de la construcción y demolición en la ciudad de 
Reciclaje de EAFD a través de la lixiviación selectiva de zinc con soluciones de citrato: comparación con un ácido inorgánico

Borda ., J. López ., F. Torres., R. y Parra ., M.

Cali: un análisis hacia su gestión, manejo y aprovechamiento. Tecnura, 19(44), 157- 170. https : //doi.org/10.14483/udistrital.jour.tecnura.2015.2.a12 个Ver página 134

[Siame et al., 2019] Siame, M. C., Kaoma, J., Hlabangana, N. y Danha, G. (2019). An attainable region approach for the recovery of iron and zinc from electric arc furnace dust.South African Journal of Chemical Engineering, 27, 35-42. https://doi.org/10.1016/j.sajce.2018.12.002 个Ver página 133

[Simonyan et al., 2019] Simonyan, L. M., Alpatova, A. A. y Demidova, N. V. (2019). The EAF dust chemical and phase composition research techniques. Journal of Materials Research and Technology, 8(2),1601-1607. https://doi.org/10.1016/j.jmrt.2018.11.005 个Ver página 136

[Torres y Lapidus, 2016] Torres, R. y Lapidus, G. T. (2016). Copper leaching from electronic waste for the improvement of gold recycling. Waste Management, 57, 131-139. https : / doi . org/10 . $1016 / j$.wasman.2016.03.010 个Ver página 133,134

[Torres y Lapidus, 2017] Torres, R. y Lapidus, G. T. (2017). Closed circuit recovery of copper, lead and iron from electronic waste with citrate solutions. Waste Management, 60, 561-568. https: // doi.org/10.1016/j.wasman.2016.12.001 个Ver página 134

[Walburga Keglevich de Buzin et al., 2017] Walburga Keglevich de Buzin, P. J., Heck, N. C. y Vilela, A. C. F. (2017). EAF dust: An overview on the influences of physical, chemical and mineral features in its recycling and waste incorporation routes. Journal of Materials Research and Technology, 6(2), 194-202. https://doi.org/10.1016/j.jmrt.2016.10.002个Ver página 133, 134, 136

[Xargay et al., 2019] Xargay, H., Ripani, M., Caggiano, A., Folino, P. y Martinelli, E. (2019). Uso de materiales reciclados en compuestos cementicios. Tecnura, 23(60), 38-51. https : / doi . org/ 10 . $14483 / 22487638.14697 \uparrow$ Ver página 134

[Yang et al., 2011] Yang, H., Liu, J. y Yang, J. (2011). Leaching copper from shredded particles of waste printed circuit boards. Journal of Hazardous Materials, 187(1-3), 393-400. https : / doi . org/10 . $1016 / j$.jhazmat.2011.01.051 个Ver página 142

[Zhang et al., 2011] Zhang, Y., Yu, X. y Li, X. (2011). Zinc recovery from franklinite by sulphation roasting. Hydrometallurgy, 109(3-4), 211-214. https://doi.org/10.1016/j.hydromet. $2011.07 .002 \uparrow$ Ver página 141

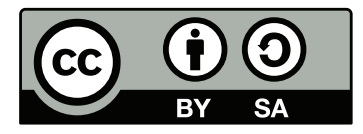

